\title{
Once a Known Veterinary Pathogen, Now a Forgotten Zoonosis. Case Report of An Invasive Staphylococcus intermedius Group Infection
}

\author{
Lucia Ines Ubiergo ${ }^{1}$ and Maximiliano Gabriel Castro (iD) ${ }^{1, *}$ \\ ${ }^{1}$ Internal Medicine Department, J.B. Iturraspe Hospital, Santa Fe, Argentina \\ Corresponding author: Internal Medicine Department, J.B. Iturraspe Hospital, Santa Fe, Argentina. Email: mgabrielcastro@outlook.com \\ Received 2020 November 15; Revised 2021 March 07; Accepted 2021 March 30.
}

\begin{abstract}
Introduction: Staphylococcus intermedius group (SIG), a known veterinary pathogen with the potential for zoonotic human infections, comprises S. intermedius, S. pseudintermedius, and S. delphini, which are not easily distinguishable. Without the proper equipment and procedures, it cannot be distinguished from Staphylococcus aureus (SAu), which causes underestimation of its true incidence.

Case Presentation: A 52-year-old male with diabetes presented with complaints of fever and malaise. He developed respiratory failure and altered mental status; hence, intensive care was provided to him. Blood cultures and bronchoalveolar lavage culture developed methicillin-resistant SIG. Despite rapid adjustment of empiric antibiotic therapy, he died of multiple organ failure.

Conclusions: Incorporating knowledge about this new pathogen and its aggressiveness into daily clinical practice can, through a high index of suspicion and detailed anamnesis, reduce misdiagnoses.
\end{abstract}

Keywords: Staphylococcus intermedius, Dog, Staphylococcus aureus, Zoonoses, Staphylococcal infections, Methicillin Resistance

\section{Introduction}

Staphylococcus intermedius group (SIG) (1), which contains the bacteria S. intermedius, S. pseudintermedius, and S. delphini (2), is a coagulase-positive catalase-positive subset of the genre Staphylococcus, that exhibits beta-hemolysis on blood agar plates (3) and is commonly isolated in dogs (4), pigeons, visons, cats, horses, foxes, racoons, and goats (5).

Although differentiation between the three pathogens is possible with equivocal microbiological algorithms that use tests such as arginine-dehydrolase and mannitol fermentation, it is mainly carried out with techniques such as matrix-assisted laser desorption ionization-time of flight mass spectrometry (MALDI-TOF MS) $(1,6)$.

At a population level, SIG presents as a rare and transient member of skin and mucosal flora in humans. However, it can be frequently identified among dog owners and veterinary practitioners (5). Moreover, it has shown a pathogenic role in skin, and soft tissue wound infections $(2,3)$ - particularly in dog bite wounds- and has recently shown potential for rare invasive infections. Nevertheless, the true incidence of SIG in human wounds is probably underestimated due to its misclassification for Staphylococcus aureus (SAu) $(2,3)$ and case reports of invasive infections -endocarditis, catheter-associated infections, pneumonia, mastoiditis, sinusitis, and brain abscesses- are scarce (5).

We aim to present a case report of an invasive presentation of this pathogen that sheds light upon its high barrier for suspicion, driven by the underrecognition of its clinical importance.

\section{Case Presentation}

A 52-year-old male rural worker in contact with little and big animals, with a diagnosis of type 2 diabetes mellitus without treatment adherence, was admitted to the hospital. He reported 5 days of fever and malaise and presented with a symmetric cutaneous rash, temporally related to $\beta$-lactam antibiotic ingestion. Later on, during hospitalization, he complained of new-onset generalized myalgias. He had a history of potential exposure to rodent excretes at his workplace, which alerted physicians of the possibility of leptospirosis.

At the Emergency Department, the patient was febrile at $38.5^{\circ} \mathrm{C}$, and his blood pressure and heart rate were $140 / 80$ $\mathrm{mmHg}$ and $110 \mathrm{bpm}$, respectively. He initially required low supplemental oxygen with an inspired fraction of oxygen

Copyright ( ) 2021, International Journal of Infection. This is an open-access article distributed under the terms of the Creative Commons Attribution-NonCommercial 4.0 International License (http://creativecommons.org/licenses/by-nc/4.0/) which permits copy and redistribute the material just in noncommercial usages, provided the original work is properly cited. 
of $28 \%$, reaching a pulse oximetry $\mathrm{SatO}_{2}$ of $96 \%$. Physical examination revealed right-lower lobe pulmonary crackles at inspiration, without further relevant findings.

Blood samples were taken for cultures and routine blood investigations, apart from HIV and leptospirosis serologies (Table 1). In addition, a chest x-ray was performed that indicated atypical community-onset pneumonia (Figure $1 \mathrm{~A}$ ), and ceftriaxone was started at a dose of $1 \mathrm{~g}$ every 12 hours.

Two days after arrival, he developed respiratory failure requiring admission to the intensive care unit (ICU) for frequent monitoring but without need for invasive mechanical ventilation (IVM) at first. At the time, he presented with bilateral pulmonary crackles, correlated to findings on a new chest x-ray (Figure $1 \mathrm{C}$ ) and a posterior chest computed tomography (Figure 1D), which showed nodules in both lungs.

The Microbiology Laboratory informed bacterial growth in both blood culture bottles within 10 hours of incubation in a BacT/ALERT ${ }^{\odot}$ (bioMérieux, US) automated microbial identification system. After the positive result, a sample was transferred to a solid growth medium, and catalase-positive Gram-positive Staphylococci colonies were identified.

Thus, the treating physicians added vancomycin at a dose of $1 \mathrm{~g}$ every 12 hours to empiric antibiotic treatment, suspecting SAu as the etiologic bacteria. With this presumptive diagnosis, a transthoracic echocardiogram was performed, with no evidence of vegetations. Afterward, the Microbiology Laboratory identified the etiologic bacteria as S. pseudintermedius through the VITEK ${ }^{\circledR} 2$ Compact (bioMérieux, US) automated identification and antibiotic susceptibility testing system.

This result was manually confirmed with a positive pyrrolidonyl arylamidase enzyme (PYR) test as well as a positive O-nitrophenyl- $\beta$-D-galactopyranoside (ONPG) test and a negative Voges-Proskauer test. Methicillin resistance was detected with an oxacillin disk diffusion test. Due to an altered mental status and hypoxemia-driven high ventricular rate auricular fibrillation, he required orotracheal intubation for IMV during the ICU stay. In the process, mini-bronchoalveolar lavage fluid was obtained for culture, which later allowed for the diagnosis of methicillinresistant S. pseudintermedius pneumonia. Despite all provided interventions, the patient died due to multiple organ failure.

\section{Discussion}

This case report displays one of the few descriptions of invasive infection by S. pseudintermedius. This microorganism colonizes the nares and anal mucosa of healthy cats and dogs, but it is also recognized as a veterinary pathogen $(4,6)$. Moreover, SIG is recognized as an infectious agent (7), particularly in special populations -namely people of old age as well as those with diabetes or immunodepression (3).

In a retrospective case series of 81 patients with SIG infections, only $7 \%$ reported contact with dogs, allegedly due to the under-registry of epidemiological data in clinical files. In $60 \%$ of investigated cultures in such series, the result was polymicrobial, failing to discriminate between the clinical characteristics of those patients with monomicrobial cultures versus those with polymicrobial cultures. Therefore, the clinical importance of SIG infections is unknown in polymicrobial settings (3).

Detailed clinical history with adequate epidemiological data recollection, with emphasis on occupational exposures, is key to our ability to elaborate papers of quality about these bacteria's true incidence and dominant clinical presentations.

Adding complexity to the problem, SIG and SAu, apart from sharing clinical scenarios and risk factors, share morphological similarities that derive from frequent misclassifications of one for the other. This is the case, in particular, of settings without automatic microbiological identification systems (3); or without personnel with appropriate training and standardized algorithms for microbial identification (2). Therefore, true incidence cannot be ascertained until after these obstacles have been sorted out.

On top of that, cefoxitin disk diffusion tests, which are used to evaluate methicillin resistance in SAu, provide equivocal results with SIG when compared to results obtained with oxacillin tests. Hence, either ignorance about this fact or the misclassification of one organism for the other $(3,6)$, could derive from the premature de-escalation of antibiotics and poor clinical results. It is noteworthy that, despite the relatively low reported incidence of methicillin-resistance in SIG, the specimen isolated from our patient had a positive oxacillin test, as a surrogate for methicillin, without resistance to other investigated antibiotics.

In our center, we have only had four clinical isolates of SIG throughout last year, three of which were from blood cultures and one from a breast abscess. This is probably a reflection of the low rate of the culture of communityacquired skin and soft tissue infections in our hospital 


\begin{tabular}{|c|c|c|c|}
\hline Variables & Admission to General Ward & Admission to Intensive Care Unit & $\begin{array}{c}\text { Final stage of Hospitalization in Intensive } \\
\text { Care Unit }\end{array}$ \\
\hline Hemoglobin $(\mathrm{g} / \mathrm{dL})$ & 16.8 & 14.0 & 13.8 \\
\hline White blood cells (cells/mm³) & 16.380 & 18.000 & 18.300 \\
\hline Neutrophils (\%) & 94.9 & 90.1 & 92.3 \\
\hline Platelet count (plat/L) & 201.000 & 81.000 & 42.000 \\
\hline Urea (mg/dL) & 49 & 66 & 75 \\
\hline Creatinine (mg/dL) & 0.57 & 0.46 & 2.1 \\
\hline Sodium $(\mathbf{m E q} / \mathbf{L})$ & 132 & 133 & 128 \\
\hline Potassium $(\mathbf{m E q} / \mathbf{L})$ & 4.05 & 4.71 & 5.1 \\
\hline Glycemia (mg/dL) & 316 & 219 & 310 \\
\hline $\operatorname{AST}(\mathbf{U} / \mathbf{L})$ & 60 & 57 & 81 \\
\hline $\operatorname{ALT}(\mathbf{U} / \mathbf{L})$ & 37 & 38 & 56 \\
\hline $\operatorname{ALP}(\mathbf{I U} / \mathbf{L})$ & 136 & 148 & 160 \\
\hline Total bilirubin/direct bilirubin (mg/dL) & 0.47 & 0.48 & 2.5 \\
\hline $\mathbf{C K}(\mathbf{U} / \mathbf{L})$ & 992 & - & - \\
\hline $\operatorname{Albumin}(\mathrm{g} / \mathrm{dL})$ & 2.04 & - & - \\
\hline Lactate $(\mathbf{m g} / \mathbf{d L})$ & - & 30.8 & - \\
\hline pH & - & 7.36 & 7.26 \\
\hline $\mathrm{pCO}_{2}(\mathrm{mmHg})$ & - & 35.5 & 53.9 \\
\hline $\mathrm{pO}_{2}(\mathrm{mmHg})$ & - & 98 & 62.7 \\
\hline $\mathrm{HCO}^{3-}(\mathrm{mmHg})$ & - & 19.8 & 23.9 \\
\hline Supplemental oxygen & $\mathrm{FiO}_{2} 28 \%$ & $\mathrm{FiO}_{2} 50 \%$ & Mechanical ventilation in prone position \\
\hline Serologies & \multicolumn{3}{|c|}{ Hantavirus: negative; Leptospirosis: negative; HIV: negative; Syphilis: negative; Hepatitis B: negative } \\
\hline Ecocardiography & \multicolumn{3}{|c|}{ Slight increase in left atrium diameter; No vegetations; Ejection fraction $65 \%$; Grade I mitral insufficiency } \\
\hline
\end{tabular}

since most SIG isolates reported in the literature are from such samples $(1,3)$. In the case of our patient, the initial clinical diagnosis was that of community-acquired pneumonia. However, the epidemiological suspicion of leptospirosis, due to the contact of the patient with rodent excretes and the fact that this infection is endemic in our region, justified its investigation, although it was subsequently negative.

After that, complementary examinations, Gram stain results, and the torpid course of illness raised the suspicion of SAu bacteriemia; therefore, led us to adjust the empiric antibiotic therapy.

Although SIG pneumonia complicated with bacteriemia, which was the final diagnosis, never occurred to us in the list of differential diagnoses, the high standards of care of our Microbiology Laboratory and its fast results permitted the prompt arrival at such diagnosis. Nevertheless, it remains a question whether the first 48 hours without an appropriate antibiotic therapy was determinant for the patient's demise, or if the course of the disease was marked solely by the pathogen's aggressiveness.

\subsection{Conclusions}

Incorporating and producing knowledge about SIG infections, which can resemble SAu infections clinically and microbiologically, together with an exhaustive anamnesis, including detailed data about patients' occupational and non-occupational exposures, will allow for fewer diagnostic mistakes involving this pathogen, therefore isolating SIG incidence from that of SAu. 

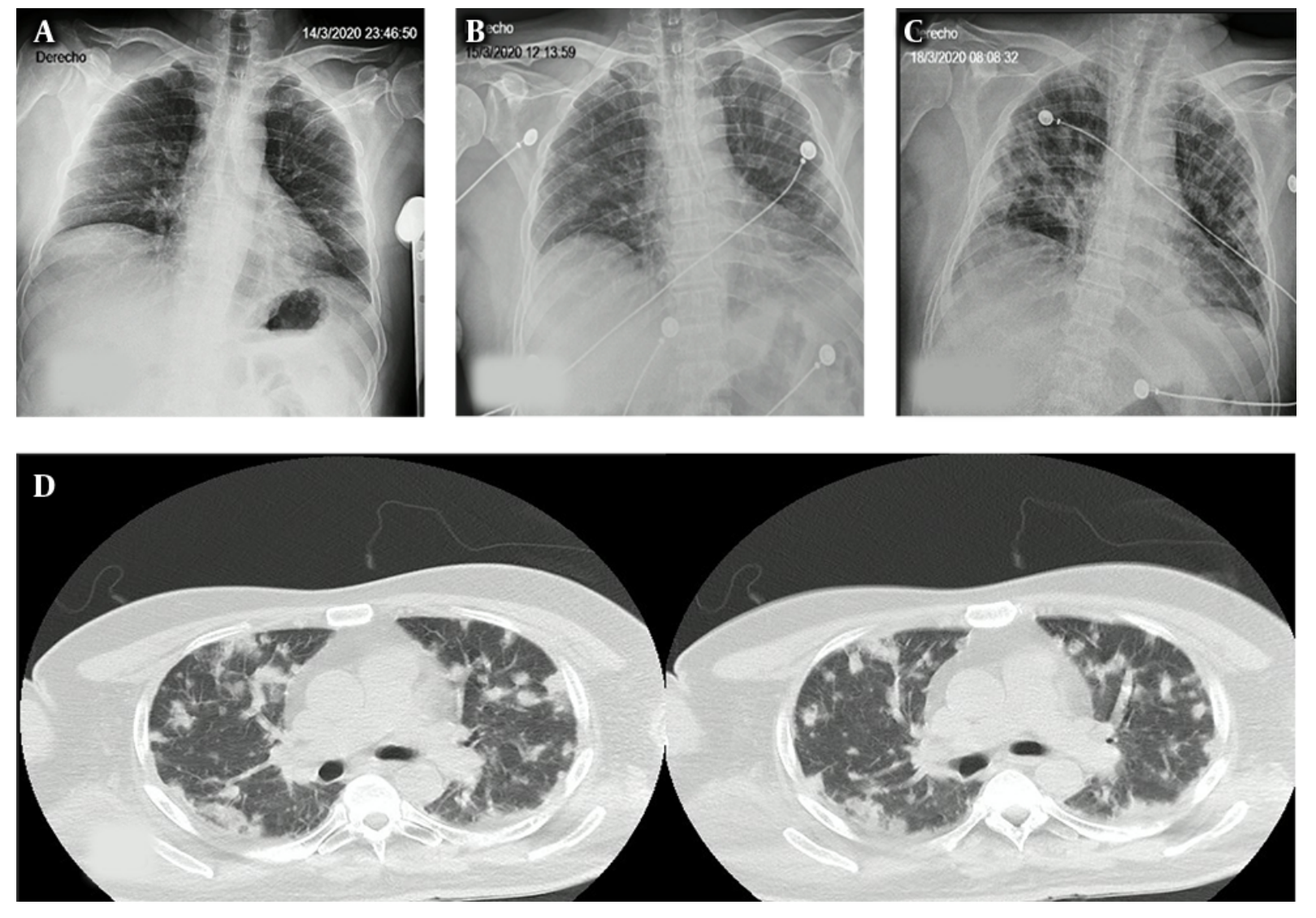

Figure 1. A, chest X-Rey at admission with interstitial infiltrates; B, chest X-Rey with accentuation of interstitial infiltrates; C, chest X-Rey at ICU admission with new bilateral alveolar infiltrates; $\mathrm{D}$, chest $\mathrm{CT}$ with nodular bilateral infiltrates, along with areas of consolidation.

\section{Footnotes}

Authors' Contribution: Both authors were part of the medical team in charge of the patient and participated in the conception of the case report. L.U. took charge of acquiring a copy of the clinical files, chest X-ray and CT. They both reviewed the literature and drafted the document, while M. C. revised the paper. After the final version of the document was ready, they approved it and agreed to the accountability of the presented paper's accuracy and integrity.

Conflict of Interests: The authors declare no conflict of interest.

Ethical Approval: The case report was approved by the hospitals authorities, which provided access to the patients files, and its ethical board. Due to the nature of this article, they did not produce an ethical approval code.

Funding/Support: The article needed no funding.

Informed Consent: The patient's family gave express consent to the publication of this document and to the us- age of his medical files, provided that his identity was preserved

\section{References}

1. Viau R, Hujer AM, Hujer KM, Bonomo RA, Jump RL. Are Staphylococcus intermedius infections in humans cases of mistaken identity? A case series and literature review. Open Forum Infect Dis. 2015;2(3):ofv110. doi: 10.1093/ofid/ofv110. [PubMed: 26509181]. [PubMed Central: PMC4536328].

2. Lee J, Murray A, Bendall R, Gaze W, Zhang L, Vos M. Improved detection of Staphylococcus intermedius group in a routine diagnostic laboratory.J Clin Microbiol. 2015;53(3):961-3. doi: 10.1128/JCM.02474-14. [PubMed: 25502532]. [PubMed Central: PMC4390641].

3. Yarbrough ML, Lainhart W, Burnham CA. Epidemiology, clinical characteristics, and antimicrobial susceptibility profiles of human clinical isolates of Staphylococcus intermedius group. J Clin Microbiol. 2018;56(3). doi: 10.1128/JCM.01788-17. [PubMed: 29305548]. [PubMed Central: PMC5824035].

4. Talan DA, Goldstein EJ, Staatz D, Overturf GD. Staphylococcus intermedius: Clinical presentation of a new human dog bite pathogen. Ann Emerg Med. 1989;18(4):410-3. doi: 10.1016/s0196-0644(89)80582-7. [PubMed: 2705672]. 
5. Bond R, Loeffler A. What's happened to Staphylococcus intermedius? Taxonomic revision and emergence of multi-drug resistance. $J$ Small Anim Pract. 2012;53(3):147-54. doi: 10.1111/j.1748-5827.2011.01165.x. [PubMed: 22251285].

6. Wu MT, Burnham CA, Westblade LF, Dien Bard J, Lawhon SD, Wallace MA, et al. Evaluation of oxacillin and cefoxitin disk and mic breakpoints for prediction of methicillin resistance in human and veterinary isolates of Staphylococcus intermedius group. J Clin Microbiol.
2016;54(3):535-42. doi: 10.1128/JCM.02864-15. [PubMed: 26607988]. [PubMed Central: PMC4767974].

7. Somayaji R, Priyantha MA, Rubin JE, Church D. Human infections due to Staphylococcus pseudintermedius, an emerging zoonosis of canine origin: report of 24 cases. Diagn Microbiol Infect Dis. 2016;85(4):471-6. doi: 10.1016/j.diagmicrobio.2016.05.008. [PubMed: 27241371]. 